\title{
Crystal Structure of Gramicidin S Hydrochloride at 1.1 Å Resolution
}

\author{
Akiko AsANo and Mitsunobu Dor ${ }^{\dagger}$ \\ Osaka University of Pharmaceutical Sciences, Nasahara, Takatsuki, Osaka 569-1094, Japan
}

\begin{abstract}
Gramicidin S hydrochloride [cyclic(-Val-Orn-Leu-D-Phe-Pro-) $)_{2} \cdot 2 \mathrm{HCl}$ ] was crystallized in the space group $P 22_{1} 2{ }_{1}$ with cell parameters $a=34.988(1) \AA, b=23.381(1) \AA, c=18.8662(5) \AA, V=15433(1) \AA^{3}$ and $Z=8$. The diffraction data were collected at $1.1 \AA$ resolution. The structure refinements converged at $R=0.165$. Two independent peptide molecules were found, which formed antiparallel-sheets having intramolecular hydrogen bonds between Val and Leu residues. The peptides turned at the D-Phe-Pro moieties, and the conformations were defined as type II' $\beta$-turn. Adjacent molecules formed an angle of $49^{\circ}$ and were arranged side-by-side to form a $\beta$-sheet along the $c$-axis.
\end{abstract}

(Received October 3, 2018; Accepted November 13, 2018; Published on web January 10, 2019)

Gramicidin S (GS) is an antibiotic cyclic decapeptide [cyclo(-ValOrn-Leu-D-Phe-Pro- $)_{2}$ ] that forms a stable $\beta$-turn and antiparallel $\beta$-sheet (Fig. 1). ${ }^{1}$ This enables GS to be utilized as a scaffold for studies of turn and sheet structures. ${ }^{1,2}$ Although many GS analogues have been studied, there have been few reports on the crystal structures of GS. This is likely because the crystals of many GS analogues diffract X-rays at low resolution, making structural analysis difficult. A preliminary X-ray study was reported by Hong et al. in 1957.3 The first structure was reported for the GS-urea complex, ${ }^{4,5}$ and then the Orn-protected analogues were solved. 6 More recently, a double-stranded helical twisted $\beta$-sheet was revealed in unprotected GS (trifluoroacetic acid form, GS.2TFA) crystals. ${ }^{8}$

In the present study, the structure of the conventional hydrochloride form of GS (GS.2HCl) is reported (Table 1). Crystals of $\mathrm{GS} \cdot 2 \mathrm{HCl}$ were grown from a $60 \%$ methanol solution, and the diffractions were measured at $1.1 \AA$ resolution. The structure was solved using the dual-space recycling method with SHELXD. ${ }^{9}$ Two independent molecules (molecules A and B) were found in a symmetric unit (Fig. 2). Disordered chlorine ions were detected at five sites. In addition, two water molecules were located, though unknown weak peaks (lower than $0.881 \mathrm{e}^{-3}$ ) remained in solvent regions. These weak peaks appear to be caused by diffractions having a low mean of $I / \sigma$ over $1.4 \AA$ resolution $(<10)$ and disordered solvent. They are not included in the refinements so as to avoid conflicts in the hydrogen-bond geometries. The structure ultimately converged at $R=0.1650$. The crystallographic achievement do not meet the standard criteria of the Cambridge Crystallographic Data Center. Nonetheless, 61 years after it was first reported, the structure of $\mathrm{GS} \cdot 2 \mathrm{HCl}$ remains an interesting peptide with which to study $\beta$-turn and sheet structures.

The peptide molecules form an antiparallel $\beta$-sheet structure with the following intramolecular hydrogen bonds: $\mathrm{Val}^{1-}$ $\mathrm{NH} \cdots \mathrm{O}=\mathrm{Leu}^{8}, \quad \mathrm{Leu}^{3}-\mathrm{NH} \cdots \mathrm{O}=\mathrm{Val}^{6}, \quad \mathrm{Val}^{6}-\mathrm{NH} \cdots \mathrm{O}=\mathrm{Leu}^{3}$ and $\mathrm{Leu}^{8}-$ $\mathrm{NH} \cdot \cdots \mathrm{O}=\mathrm{Val}^{1}{ }^{1}$ (Table S1). The N61B...O32B distance is somewhat long for a hydrogen bond, but it is included in the table and figures. The backbone conformations of molecules $\mathrm{A}$ and $\mathrm{B}$ are similar to each other $(\mathrm{RMS}=0.404 \AA$, Fig. S1), with differences

† To whom correspondence should be addressed.

E-mail: doit@gly.oups.ac.jp mainly in the side-chain orientations. The peptide rings turn at two D-Phe-Pro moieties. From the selected torsion angles of the $i+1$ and $i+2$ positions, the turns are defined as type II' $\beta$-turn (Table 2). These characteristics are commonly observed in the structures of GS analogues. The conformational differences are observed in the Orn side chains. The Orn side chains of molecule A (Orn-A) extend into the solvent region (Fig. S2), and no intramolecular hydrogen bonds are formed. The $\varepsilon$-amino groups of Orn-A interact with chlorine ions and solvent molecules. The extended conformations of the Orn side chain have been observed in the GS-urea complex. In contrast, the Orn side chains of molecule B (Orn-B) are folded into the peptide ring, and the N25B and N75B atoms ( $\varepsilon$-amino groups of Orn) form hydrogen bonds with $\mathrm{O}=\mathrm{D}-\mathrm{Phe}$ (Table $\mathrm{S} 1$ ). This type of intramolecular hydrogen bond is often observed in the Orn side chains of GS analogues.

The peptide molecules are arranged side-by-side and form $\beta$-sheet structures along the $c$-axis (Fig. 3). Three intermolecular

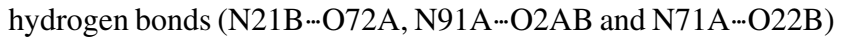
are formed. The symmetry-translated molecules also form hydrogen bonds at N41A-..O72B and N91B-..O22A (Table S1). Adjacent molecules $\mathrm{A}$ and $\mathrm{B}$ form an angle of $49^{\circ}$ between them, but the $\beta$-sheet formed does not create the helical twisted $\beta$-sheet channels observed in GS.2TFA. The molecular packing

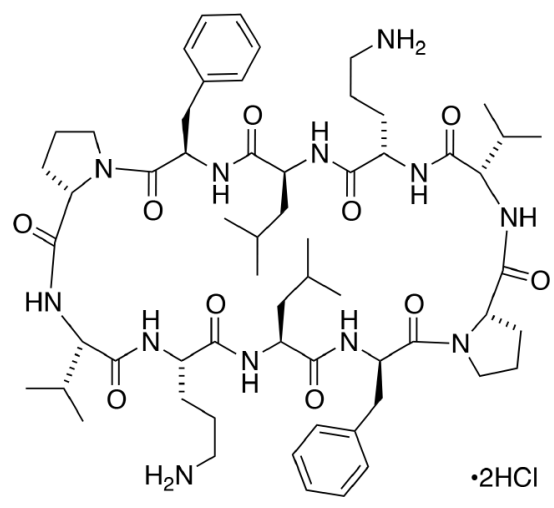

Fig. 1 Chemical structure of gramicidin S hydrochloride. 
Table 1 Crystal and experimental data

Chemical formula: $2\left(\mathrm{C}_{60} \mathrm{H}_{94} \mathrm{~N}_{12} \mathrm{O}_{10} \mathrm{Cl}_{2}\right) \cdot 2 \mathrm{H}_{2} \mathrm{O}$

Formula weight $=2460.7$

$T=93 \mathrm{~K}$

Crystal system: orthorhombic $\quad$ Space group: $P 22_{1} 2$

$a=34.988(1) \AA$

$b=23.381(1) \AA$

$c=18.8662(5) \AA$

$V=15433(1) \AA^{3}$

$Z=8$

$D_{\mathrm{X}}=1.059 \mathrm{~g} \mathrm{~cm}^{-3}$

Radiation: $\mathrm{Cu} K \alpha(\lambda=1.54184 \AA)$

$\mu(\mathrm{Cu} K \alpha)=1.211 \mathrm{~mm}^{-1} \quad F\left(\begin{array}{lll}0 & 0 & 0)=5280\end{array}\right.$

Crystal size $=0.10 \times 0.16 \times 0.52 \mathrm{~mm}^{3}$

No. of reflections collected $=28227$

No. of independent reflections $=11453$

No. of reflections used $[I>2 \sigma(I)]=9329$

Data/Restraints/Parameters $=9329 / 1063 / 1490$

$\theta$ range for data collection: 2.27 to $44.49^{\circ}$ (1.1 Å resolution)

Goodness-of-fit $=2.756$

$R$ indices $[I>2 \sigma(I)]: R 1=0.1650, w R 2=0.4007$

$R$ indices (all data): $R 1=0.1793, w R 2=0.4081$

$(\Delta / \sigma)_{\max }=0.024$

$(\Delta \rho)_{\max }=0.881 \mathrm{e}^{-3} \quad(\Delta \rho)_{\min }=-0.561 \mathrm{e}^{-3}$

Measurement: Rigaku Xtalab P200

Structure determination: SHELXD ${ }^{9}$

Refinement: SHELXL ${ }^{10}$

CCDC deposition number: 1870209

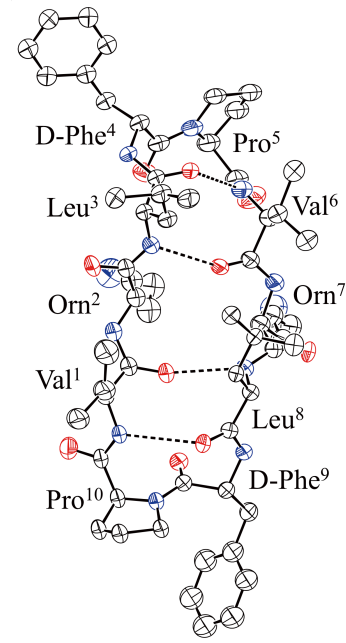

A

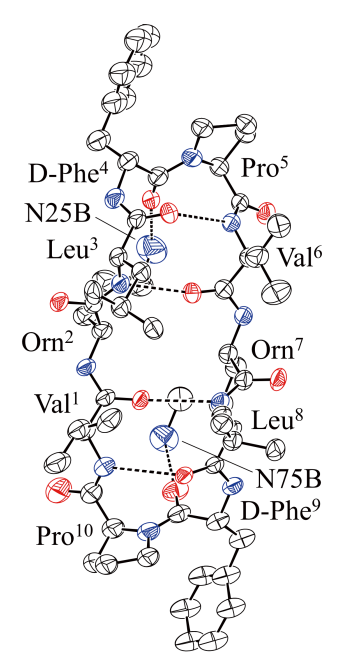

B
Fig. 2 ORTEP views of the title compound. Molecules A and B are found in an asymmetric unit. Hydrogen atoms are omitted for clarity. Dotted lines show hydrogen bonds. Residues are labeled with their names. Intramolecular hydrogen bonds involving Orn side chains are found in molecule $\mathrm{B}$, and the $\mathrm{N} 25 \mathrm{~B}$ and $\mathrm{N} 75 \mathrm{~B}$ atoms are labeled. Displacement ellipsoids are drawn at the $30 \%$ probability level.

of $\mathrm{GS} \cdot 2 \mathrm{HCl}$ creates hydrophilic and hydrophobic regions within the crystal (Fig. S4). The Orn side chains result in the hydrophilic regions, where the chlorine ions and water molecules are distributed. The Val and Leu side chains are orientated toward the other side of the peptide. The crystal packing leads to crowding of these aliphatic side chains, which results in the formation of hydrophobic regions. The amphiphilicity of GS causes the characteristic molecular packing in the crystal.
Table 2 Selected torsion angles $\left({ }^{\circ}\right)$ at the turn position (D-PhePro)

\begin{tabular}{crrrrr}
\hline \multirow{2}{*}{ Position } & \multicolumn{2}{c}{ Molecule A } & & \multicolumn{2}{c}{ Molecule B } \\
\cline { 2 - 3 } \cline { 5 - 6 } & \multicolumn{1}{c}{$4-5$} & $9-10$ & & \multicolumn{1}{c}{$4-5$} & $9-10$ \\
\hline$\phi_{i+1}$ & $61(3)$ & $57(3)$ & & $51(3)$ & $67(3)$ \\
$\varphi_{i+1}$ & $-130(2)$ & $-134(2)$ & & $-122(3)$ & $-132(2)$ \\
$\phi_{i+2}$ & $-69(3)$ & $-87(3)$ & & $-77(4)$ & $-81(3)$ \\
$\varphi_{i+2}$ & $-11(4)$ & $-2(4)$ & & $-1(5)$ & $2(3)$ \\
\hline
\end{tabular}

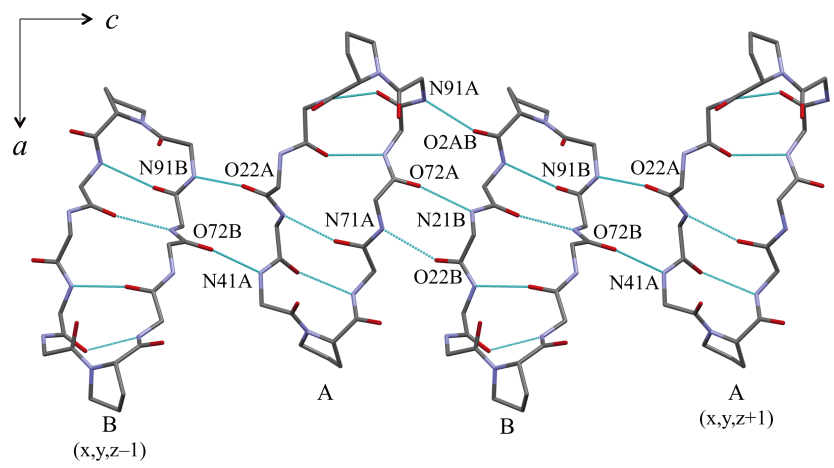

Fig. 3 Packing diagram. Only the backbones are drawn for clarity. $\mathrm{A} \beta$-sheet structure is formed by adjacent molecules along the $c$-axis. The angle between the peptide rings of molecules A and B is $49^{\circ}$. Skyblue lines show hydrogen bonds. Atoms related to intermolecular hydrogen bonds are labeled.

\section{Supporting Information}

A CIF format file, Table S1 and Figs. S1 - S4. These materials are available free of charge on the Web at http://www.jsac.or.jp/ xraystruct/.

\section{References}

1. N. Izumiya, T. Kato, H. Aoyagi, M. Waki, and M. Kondo, "Synthetic Aspects of Biologically Active Cyclic PeptidesGramicidin $S$ and Tyrocidines", 1979, Tokyo, Kodansha.

2. M. Tamaki, I. Sasaki, M. Kokuno, M. Shindo, and Y. Uchida, Org. Biomol. Chem., 2010, 8181, 1791.

3. D. C. Hodgkin and B. M. Oughton, Biochem. J., 1957, 65, 752.

4. S. E. Hull, R. Karlsson, P. Main, M. M. Woolfson, and E. J. Dodson, Nature, 1978, 275, 206.

5. E. Dodson, Y. Yo, Y. Yoi, and E. Ccp, Acta Crystallogr. Sect. D, 1997, D53, 151.

6. M. Doi, S. Fujita, Y. Katsuya, M. Sasaki, T. Taniguchi, and H. Hasegawa, Arch. Biochem. Biophys., 2001, 395, 85.

7. K. Yamada, M. Unno, K. Kobayashi, H. Oku, H. Yamamura, S. Araki, H. Matsumoto, R. Katakai, and M. Kawai, J. Am. Chem. Soc., 2002, 124, 12684.

8. A. L. Llamas-Saiz, G. M. Grotenbreg, M. Overhand, and M. J. van Raaij, Acta Cryst., 2007, D63, 401.

9. G. M. Sheldrick and R. O. Gould, Acta Cryst., 1995, B51, 423.

10. G. M. Sheldrick, Acta Cryst., 2015, C71, 3. 